\title{
Ancha Srinivasan (ed): Handbook of precision agriculture. Principles and applications
}

\author{
The Haworth Press Inc., New York, 683 pp, ISBN-13: 978-1-56022-945-4
}

\author{
Anton J. Haverkort
}

Received: 19 December 2006 / Accepted: 28 December 2006 / Published online: 23 January 2007

(C) Springer Science+Business Media B.V. 2007

Dr. Ancha Srinivasan is a principal researcher and manager at the Institute for Global Environmental Strategies (IGES) in Hayama, Japan. He worked in research projects in sustainable agriculture, information systems and the environment and the adaptation to climate change in agriculture. In his foreword dr. M.S. Swaminathan observes that the world is witnessing two cultures of agriculture: farming in industrialized countries is becoming agri-business supported by heavy inputs whereas in developing countries it is a primary means to livelihood. He states that precision farming helps to reduce cost while increasing productivity when designed for each cropping system in a location specific manner.

The editor defines Precision Agriculture as a holistic and environmentally friendly strategy in which farmers can vary input use and cultivation methods - including application of seeds, fertilisers, pesticides and water, variety selection, planting, tillage, harvesting - to match varying soil and crop conditions across a field. There are more definitions and they all suggest there are at least three elements critical for successful precision

A. J. Haverkort $(\square)$

Plant research International,

Wageningen University and Research Centre,

16, Wageningen, $6700 \mathrm{AA}$, The Netherlands

e-mail: anton.haverkort@wur.nl agriculture: information, technology and management.

The book contains 22 chapters to which fifty authors working at universities and institutions from all over the world contributed. It is divided in three parts. Part I deals with the principles, technologies and management issues of precision agriculture. Its basics are explained and how site specificity and information (acquired by sensors of crop, weather and soil parameters) technology converge in Global Positioning Systems (GPS). The information is stored and assembled for interpretation to be used in decision support systems. After this introduction subsequent chapters treat the site specific management of nutrients, water, control of weeds, nematodes and diseases. Here the use of appropriate sampling and statistical techniques are highlighted to ensure the drawing of representative charts of individual fields. Also sensing of crops (mostly reflection of solar radiation at various wavelengths) with handheld, tractor mounted, or carried by aeroplane or satellite is illustrated as well as maps of yields of crops within fields. Soil techniques include electric conductivity and background radiation. The resulting spatial variability is visualised in a series of full colour maps in a central gallery of the book.

Part II illustrates applications in crops and cropping systems. Here site specific management of crops such as rice, corn, soybean, cotton, potato, sugarbeet and vineyards is highlighted. In 
certain crops the spatial variation of diseases and pests (e.g. late blight and nematodes in potato) is most important to take into account whereas in other crops (e.g. sugarbeet) spatial distribution of nitrogen in the soil is addressed. Part III dedicates chapters to the current status of precision farming in Africa, Asia, Australia, Europe, Argentina and Brazil. The editor apparently has not found a reviewer to highlight the developments in North America.

The second part of the book concludes with a chapter on site specific management from a cropping system perspective. It ends stating "Many producers and scientists are frustrated by the difficulty in converting information into knowledge". It also states that economic and wide-scale adoption of site-specific management practises will only be realized when costs are minimal, we can accurately predict the impact of management options on yield and when we can transfer decision tools to end-users.

The book contains a wealth of information and will certainly be very much of use to scientists and technicians involved in spatial precision farming. Temporal precision farming (when to apply which dose) is not treated. The book gives a thorough state of art of the gadgets and gizmos but does not give a good direction of interpreting information into right decisions. If a sensor detects that there is a relative lack of vegetation should a grower then apply more or less nitrogen is only known if we know if reduced growth was due to lack of nitrogen and not due to lack of water. The site specific planting of different varieties would be of interest but is not treated, nor is the specific breeding needs for site specific farming. 